\title{
Y-junctions based on circular depressed-cladding waveguides fabricated with femtosecond pulses in Nd:YAG crystal: A route to integrate complex photonic circuits in crystals
}

\author{
Javier G. Ajates a, b, Carolina Romero a, Gabriel R. Castillo a, Feng Chen ${ }^{\text {c, }}$ \\ Javier R. Vázquez de Aldana a, * \\ a Aplicaciones del Láser y Fotónica, University of Salamanca, Pl. La Merced SN, 37008, Salamanca, Spain \\ b Spanish Center for Pulsed Lasers, M5 Bldg. Science Park, Villamayor, Salamanca, Spain \\ c School of Physics, Shandong University, 250100, Jinan, Shandong, China
}

\section{A R T I C L E I N F O}

\section{Article history:}

Received 20 April 2017

Received in revised form

5 June 2017

Accepted 6 June 2017

Available online 13 June 2017

\section{Keywords:}

Laser materials processing

Waveguides channeled

Integrated photonic devices

Crystalline materials

\begin{abstract}
A B S T R A C T
We have designed and fabricated photonic structures such as, Y-junctions (one of the basic building blocks for construction any integrated photonic devices) and Mach-Zehnder interferometers, based on circular depressed-cladding waveguides by direct femtosecond laser irradiation in Nd:YAG crystal. The waveguides were optically characterized at $633 \mathrm{~nm}$, showing nearly mono-modal behaviour for the selected waveguide radius $(9 \mu \mathrm{m})$. The effect of the splitting angle in the Y structures was investigated finding a good preservation of the modal profiles up to more than $2^{\circ}$, with $1 \mathrm{~dB}$ of additional losses in comparison with straight waveguides. The dependence with polarization of these splitters keeps in a reasonable low level. Our designs pave the way for the fabrication of arbitrarily complex 3D photonic circuits in crystals with cladding waveguides.
\end{abstract}

(C) 2017 Elsevier B.V. All rights reserved.

\section{Introduction}

Optical waveguides are the basic elements to construct complex photonic circuits [1]. The technique of direct femtosecond laser inscription has allowed the integration of compact photonic elements in transparent dielectrics [2,3] (glasses, ceramics, crystals or polymers) thus exploiting the special properties of each material. Among them, crystalline materials are very attractive due to their large transparency range, the non-linear properties, the birefringence and the optimum spectroscopic features. However, the fabrication of optical waveguides in crystals [4] is relatively difficult mainly due to the impossibility to produce, in most of them, refractive index increases localized in the region directly irradiated with the femtosecond laser. In such cases, other strategies have been developed making use of severe damage tracks produced in the crystal at higher pulse energies, as depressed-cladding waveguides [5] or stress-induced waveguides [6].

Depressed-cladding waveguides based on severe damage modifications consist of many parallel tracks inscribed in the crystal

\footnotetext{
* Corresponding author.

E-mail address: jrval@usal.es (J.R. Vázquez de Aldana).
}

forming a modified cladding and a central unexposed core [5] where light propagates through. The refractive index typically decreases at the damaged regions [7], and thus the structure is essentially a core with the same index as the bulk, surrounded by a cladding with lower index [8]. Residual stress may appear at the waveguide core $[9,10]$ due to the cumulative effect of large number of laser impacts, but it is minimized by the choice of proper fabrication parameters. In principle, the refractive index configuration of cladding waveguides supports only leaky modes [11] what means that only continuum radiation modes exist. However, when the width of the lower-index region (depressed cladding) is large, propagating modes that are very similar to confined modes are supported [12]. With the purpose of getting a good optical performance, the separation between damage tracks must be very small (typically 2-3 $\mu \mathrm{m}$ ) and the scanning velocity large enough to minimize the stress induced in the surroundings of the tracks.

In comparison with the simple double line approach [13], the main advantages of femtosecond laser written depressed-cladding waveguides are the following. Firstly, the waveguide core can be designed and fabricated with arbitrary shape and size. This is particularly important to minimize the losses due to coupling with external sources, such as optical fibers: to this end, the circular 
shape of the waveguides is the most favorable. Secondly, both TE as TM polarizations may be confined in the waveguides [14] with low polarization dependence. In addition, they share advantages such as the preservation, in the waveguide core, of the spectroscopic and non-linear properties of the bulk material [15], so that they can be fully exploited for many active devices. Another common point is the thermal stability of these waveguides up to moderate temperatures: in fact, a thermal annealing may improve the performance of the waveguides [16].

Depressed-cladding waveguides have been successfully inscribed in a large number of crystalline materials and ceramics (see Ref. [4] and references therein) and different integrated devices has been demonstrated based on these waveguides, such as compact lasers [9] or frequency converters [17]. However, the fabrication of the elements required for complex photonic circuits, such as Y beam-splitters, beam-combiners and low-loss bends, are considerably difficult to design in the basis of cladding waveguides. Recently, some advances have been done and planar Y-junctions [18-20] and Mach-Zehnder interferometers [21] have been demonstrated, all of them based on rectangular-shape cladding waveguides. However, to our knowledge, no attempt has been done in fabricating such devices with circular-shape cladding waveguides, much more difficult to implement, that are necessary in order to keep the modal profiles along a complex photonic circuit, and are more convenient in terms of coupling with external elements.

In this work we have developed an efficient model for the inscription, by direct femtosecond laser irradiation, of Y-junctions (one of the basic building blocks for construction any integrated photonic devices) based on circular depressed-cladding waveguides. Y-junctions are basic elements required for the fabrication of more complex photonic circuits, such as Mach-Zehnder interferometers, that have been also successfully implemented. The devices have been inscribed on Nd:YAG crystal, that is one of the most widely used gain media for solid-state lasers due to its outstanding fluorescence, thermal and mechanical properties. Previous work has demonstrated the possibility to fabricate waveguides based on type-I modification for operation in the midIR [22] as well as double line waveguides [23]. Y-junctions based on rectangular cladding waveguides have been fabricated previously in the surface of Nd:YAG crystal $[18,20]$. But to our knowledge, neither buried Y-junctions nor more complex devices such as interferometers, have been previously reported. We have firstly optimized the design of straight waveguides for an operation wavelength of $633 \mathrm{~nm}$. Then, Y-junctions with different splitting angles have been fabricated and optically characterized, measuring losses and polarization dependence. Finally, a Mach-Zehnder interferometer has been also implemented and studied. YAG crystals have no electro-optic properties and thus, to our knowledge, the application to sensing of such fabricated device is limited. However, the possibility to efficiently split a beam and combine it again in a single device integrated in laser crystal could be of potential interest for reducing saturation effects in high-power pulse amplification [24]. In summary, the developed technique opens the door to the fabrication of optimized arbitrarily complex 3D devices in crystals preserving modal profiles to a very good extent.

\section{Waveguides design}

Previously to the fabrication of the waveguides, it is necessary to elaborate the numerical codes allowing the inscription of the laser damage tracks that will form the waveguide claddings. In our inscription approach (sample scanning) the codes are basically sentences for the positioning of the XYZ stage that moves the sample in the laser focus.

The first step is the design of the straight circular waveguides. The two main parameters in this case are the waveguide radius $(R)$ and the separation between tracks $(d)$. Once both parameters are fixed, we fit the circumference of radius $R$ by a $N$-sides polygon, with a side length of $d$. The vertices of the polygon are the coordinates at which the damage tracks will be inscribed along the sample (see Fig. 1 a).

The splitting region in the $Y$ structure is the most critical part of our designs and it is governed by a new parameter $\alpha$, the angle between arms. Firstly, at the beginning of the splitting region, half of the damage tracks will constitute one of the arms, and the other half will constitute the other. Then, each set of tracks will change their initial direction to $\pm \alpha / 2$. Additionally, new tracks must be added as the two arms get separated in order to complete the cladding of both structures, until both arms are finally split (see Fig. $1 \mathrm{~b}$ ). We have used the design software tools AutoCAD and SolidWorks in order to visualize this parts and to check the correctness of the developed codes. The length of the splitting region depends obviously on the angle between arms $(\alpha)$ and on the radius of the waveguide $(R)$. a)

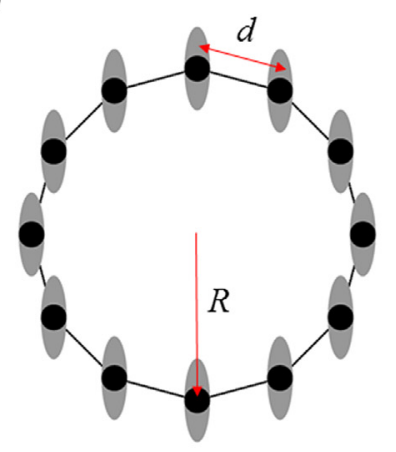

b)

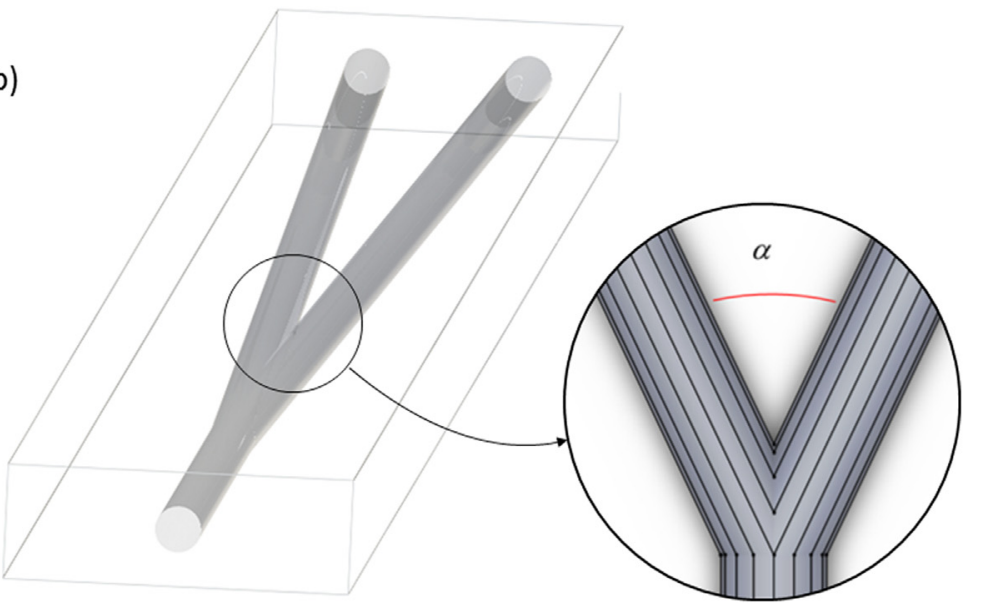

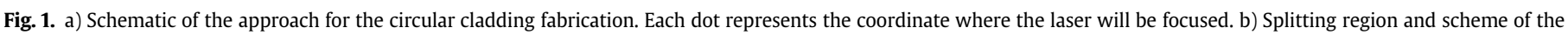
cladding tracks. 

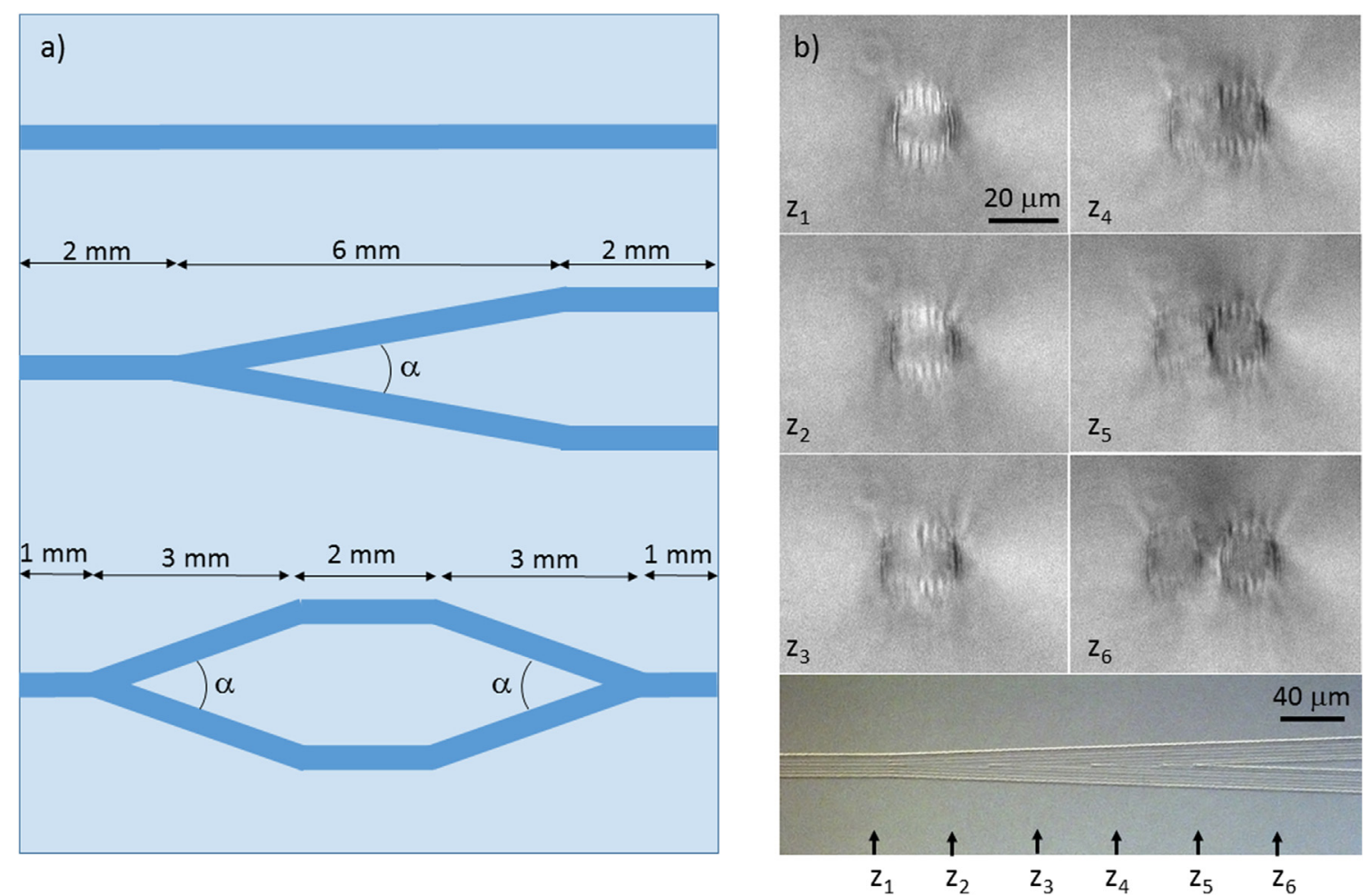

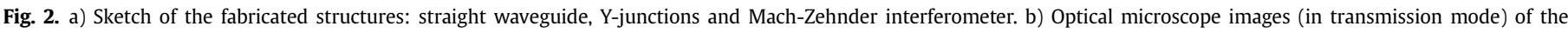

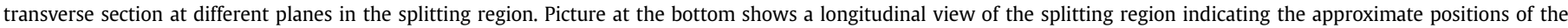
images above.

\section{Fabrication by direct femtosecond laser writing}

A Ti:Sapphire laser system with a regenerative amplifier (Spitfire, Spectra-Physics) was used for the inscription of the designed structures. The system produces linearly-polarized pulses of $\sim 120 \mathrm{fs}$ duration (Fourier limited), with a repetition rate of $1 \mathrm{kHz}$ at a central wavelength of $795 \mathrm{~nm}$. A calibrated neutral density filter, a motorized half-wave plate and a linear polarizer were used to precisely control the value of the pulse energy. The sample, an optically polished Nd:YAG ( $1 \% \mathrm{Nd}$ ) crystal with dimension of $10 \times 10 \times 3 \mathrm{~mm}^{3}$, was mounted on a high-resolution three-axis motorized stage. The laser beam was focused through one of the large faces of the sample using a microscope objective $(40 \times$, N.A. $=0.65$ ). Waveguides were written along one of the $10-\mathrm{mm}$ long axes of the crystal at a constant scanning velocity of $500 \mu \mathrm{m} / \mathrm{s}$, at a depth of $\sim 200 \mu \mathrm{m}$ below the sample surface, and with a pulse energy of $0.15 \mu \mathrm{J}$. Laser polarization was kept perpendicular to the scanning direction.

The first set of fabricated structures consisted of straight waveguides with different radii, from 9 to $20 \mu \mathrm{m}$, and a separation between adjacent tracks of $d=2 \mu \mathrm{m}$. Once the waveguides were analysed, the radius of $9 \mu \mathrm{m}$ was selected for the fabrication of the complex structures due to its nearly mono-modal behaviour at $633 \mathrm{~nm}$. Then, Y-junctions were fabricated with different splitting angles $\alpha$ between arms: $1^{\circ}$ (Y1), $2^{\circ}$ (Y2) and $4^{\circ}$ (Y4). The junctions consisted on a 2-mm long straight section followed by a splitting section $(6 \mathrm{~mm})$ and a final 2-mm long straight part (see Fig. 2 a). Finally, a Mach-Zehnder (MZ) interferometer was inscribed with splitting/combining angles of $2^{\circ}$, initial/final straight sections 1 $\mathrm{mm}$ long, splitting sections 3-mm long and a 2-mm long straight section in the middle (see Fig. 2 a).

The most critical parts of the structures are obviously the splitting regions. In Fig. 2 b) we show pictures taken in the optical microscope (transmission mode) of the transverse section at different planes in the splitting region. In the picture at the bottom (longitudinal view of this section of the waveguides) we have indicated approximately the planes in which those images were taken. As it can be seen in the pictures, the initially circular waveguide broadens giving rise to the two arms of the junction by introducing new laser tracks, at it is described in Sect. 2.

\section{Optical characterization and results}

The modal profiles of the fabricated structures, straight waveguide (WG1), Y-junctions (Y1-4) and Mach-Zehnder (MZ), were analysed at $633 \mathrm{~nm}$. A He-Ne laser was focused by a $10 \times$ microscope objective at the input face of the waveguide. A half-wave plate was used to control the polarization of the incident beam. The modal profiles at the output of the waveguides (without any polarization control element) were imaged by a $20 \times$ microscope objective onto a CMOS camera (IDS uEye SE).

Firstly, we analysed the behaviour of WG1 as reference. In Fig. 3 a) we show the recorded modal profiles for the TE and TM polarizations (perpendicular and parallel to the fs-laser damage tracks respectively) of the He-Ne laser. As it can be seen in the pictures, the waveguide behaves as nearly single-mode at this wavelength, independently on the input laser polarization. In Fig. 3 b) we represent the normalized output power for all the polarization directions of the input laser $\left(0^{\circ}\right.$ corresponds to TE polarization $)$. The waveguide shows a very isotropic behaviour, with variations of transmitted power with polarization less than $11 \%$.

The propagation loss of WG1 was measured by the scatteredlight method [25]. To this end, the beam of the He-Ne laser was coupled into the waveguide, and the scattered light was imaged through the large sample surface onto another CMOS camera (IDS uEye SE) by a $\mathrm{f}=2 \mathrm{~cm}$ lens. The light was coupled into the 

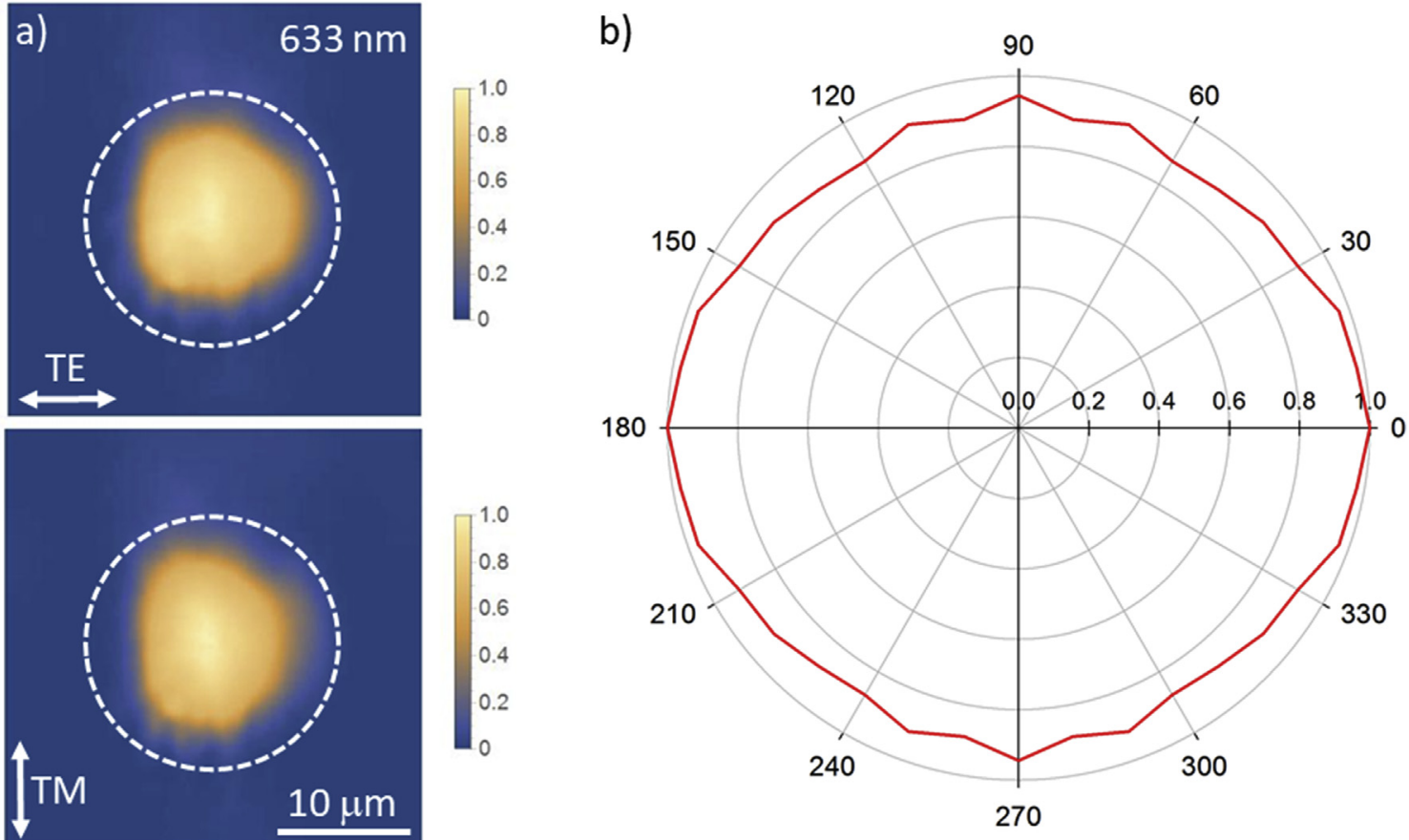

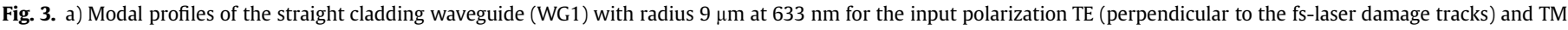

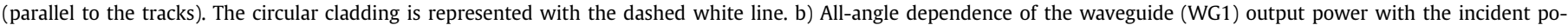
larization. $0^{\circ}$ corresponds to TE polarization. The angular resolution of the measurement is $10^{\circ}$.

waveguide several times and different independent measurements were done each time. Then, the obtained mean value of the propagation loss was $3.3 \pm 0.6 \mathrm{~dB} / \mathrm{cm}$ for both TE and TM polarizations. This value is larger than the propagation loss reported previously for circular cladding waveguides in Nd:YAG at $633 \mathrm{~nm}(1.7 \mathrm{~dB} / \mathrm{cm}$ and $2.0 \mathrm{~dB} / \mathrm{cm}$ for waveguides with $100 \mu \mathrm{m}$ - and $30 \mu \mathrm{m}$ - diameter respectively, see Ref. [26]) but due to the dependence of propagation loss with waveguide diameter, it is not surprising the value measured for our $18 \mu \mathrm{m}$ waveguides.

A similar study was done for the Y-junctions. In Fig. 4 a) we show the modal profiles at $633 \mathrm{~nm}$ for TE polarization. As it can be seen in the pictures, for $1^{\circ}$ and $2^{\circ}$ the output modes of the waveguides preserve a nearly single-mode behaviour, with a slight dependence with polarization (not shown in the figure). The splitting ratios in both cases are almost equalized (see Table 1), but they are very sensitive to the alignment of the input $633 \mathrm{~nm}$ beam.

Table 1

Splitting ratios and additional losses (see text for an explanation) of the fabricated Yjunctions at $633 \mathrm{~nm}$

\begin{tabular}{|c|c|c|c|c|}
\hline & Y-junction $1^{\circ}$ & Y-junction $2^{\circ}$ & Y-junction $4^{\circ}$ & Mach-Zehnder \\
\hline Splitting ratio & $53 / 47$ & $52 / 48$ & $54 / 46$ & - \\
\hline \multicolumn{5}{|c|}{ Additional losses } \\
\hline $\mathrm{TE}$ & $0.2 \mathrm{~dB}$ & $1.0 \mathrm{~dB}$ & $10.2 \mathrm{~dB}$ & $2.8 \mathrm{~dB}$ \\
\hline TM & $1.0 \mathrm{~dB}$ & $1.4 \mathrm{~dB}$ & $15.4 \mathrm{~dB}$ & $5.3 \mathrm{~dB}$ \\
\hline
\end{tabular}
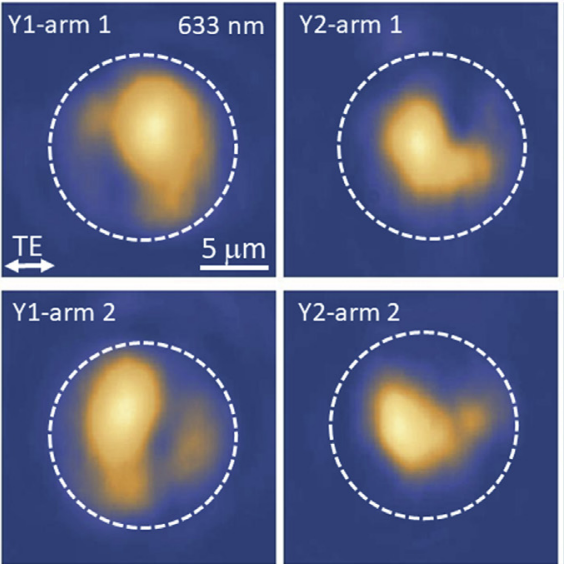
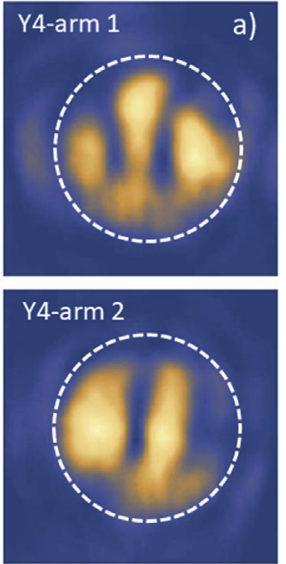

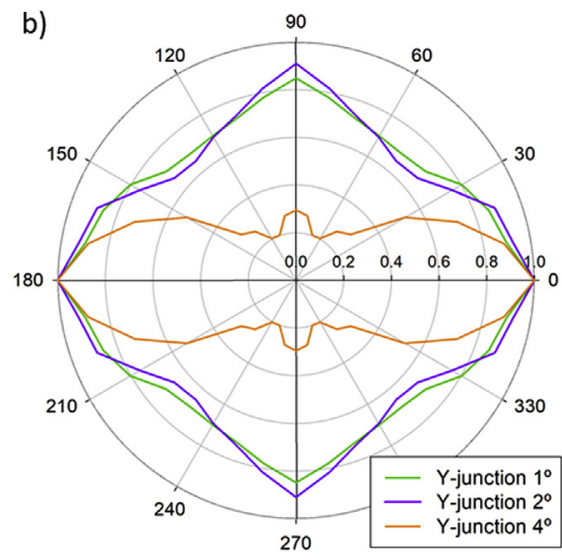

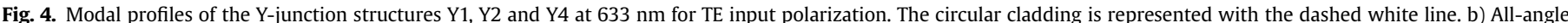

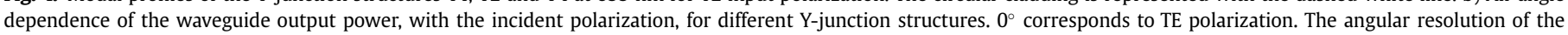
measurement is $10^{\circ}$. 

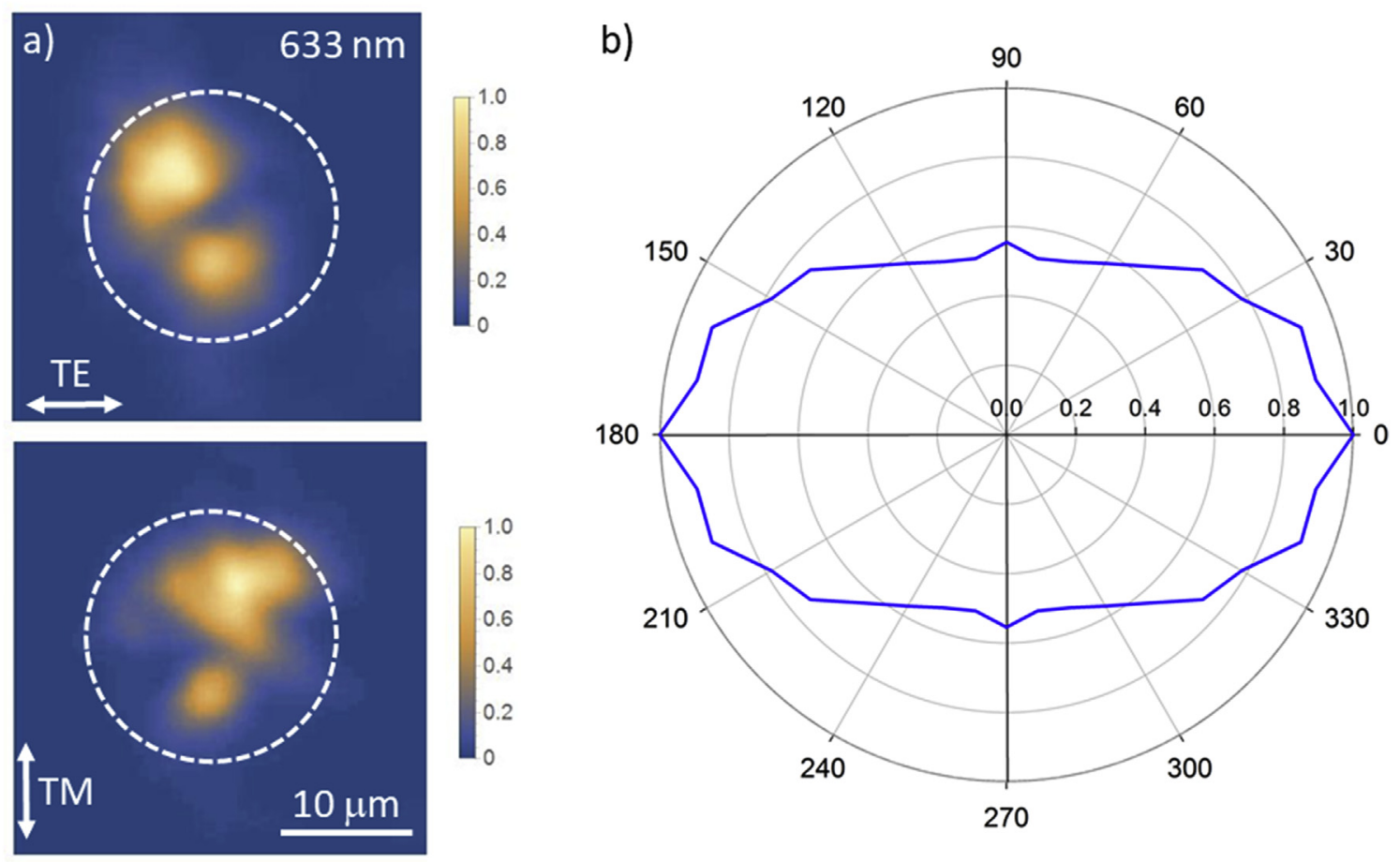

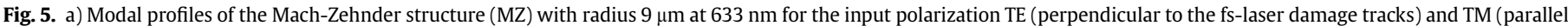

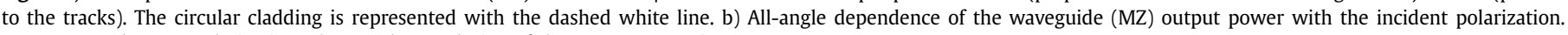
$0^{\circ}$ corresponds to TE polarization. The angular resolution of the measurement is $10^{\circ}$.

In the case of the Y-junction with $4^{\circ}$ between arms, the behaviour becomes clearly worse: higher-order modes are excited, thus degrading the modal profiles.

The losses of the Y-junctions were evaluated in the following way. We measured the total output powers of the two arms $\left(P_{Y}\right)$ for each junction and we compared the obtained values with the output power of WG1 $\left(P_{0}\right)$. Then, we did an estimation of the additional losses of each structure in comparison to those of WG1:

$\eta=-10 \cdot \log _{10}\left(P_{Y / P_{0}}\right)$

The obtained values are shown in Table 1. The junctions Y1 and Y2 show very low additional losses of 0.2 and $1.0 \mathrm{~dB}$ respectively for TE polarization, that are larger for TM polarization (1.0 and $1.4 \mathrm{~dB}$, respectively). However, for Y4 the losses increase to $10.2 \mathrm{~dB}$ (TE polarization) and $15.4 \mathrm{~dB}$ (TM polarization), what is clearly linked to the multimodal behaviour of the structure at such large angle between arms.

Concerning the Mach-Zehnder interferometer, the modal profile at output exhibits a strong dependence with polarization (see Fig. 5). The losses keep in a reasonable low level that shows a good performance not only in the splitting (that is identical to that of Y2) but also in the Y-junction between both arms.

\section{Conclusions}

In summary, we have developed a technique for the fabrication of complex photonic elements in circular depressed-cladding optical waveguides inscribed in transparent dielectrics by femtosecond laser irradiation. The technique has been demonstrated in $\mathrm{Nd}$ :YAG crystal, where we have successfully fabricated Y-junctions with splitting angles between $1^{\circ}$ and $4^{\circ}$. For small angles $\left(1^{\circ}\right)$ the junctions exhibit as low as $0.2 \mathrm{~dB}$ of additional losses compared to a straight waveguide, monomodal behaviour and low dependence with polarization direction. For larger angles the losses increases to $10.2 \mathrm{~dB}\left(4^{\circ}\right)$ and the dependence with polarization increases. A Mach-Zehnder interferometer was also fabricated, demonstrating the possibility to use the developed technique to implement also efficient Y-junctions. Then, our designs pave the way for the fabrication of arbitrarily complex 3D photonic circuits with circular cladding waveguides, technique that is particularly interesting for crystals in which refractive index increases cannot be produced by femtosecond laser writing.

\section{Acknowledgements}

We acknowledge support from Junta de Castilla y León (Projects UIC016, SA046U16) and MINECO (FIS2013-44174-P, FIS2015-71933REDT).

\section{References}

[1] G. Lifante, Integrated Photonics: Fundamentals, first ed., ,Wiley, Chichester, 2003.

[2] Rafael R. Gattass, Eric Mazur, Femtosecond laser micromachining in transparent materials, Nat. Photonics 2 (4) (2008) 219-225.

[3] Debaditya Choudhury, John R. Macdonald, Ajoy K. Kar, Ultrafast laser inscription: perspectives on future integrated applications, Laser Photonics Rev. 8 (6) (2014) 827-846.

[4] Feng Chen, J. R. Vázquez de Aldana, Optical waveguides in crystalline dielectric materials produced by femtosecond-laser micromachining, Laser Photonics Rev. 8 (2) (2014) 251-275.

[5] A.G. Okhrimchuk, A.V. Shestakov, I. Khrushchev, J. Mitchell, Depressed cladding, buried waveguide laser formed in a YAG: $\mathrm{Nd}^{3+}$ crystal by femtosecond laser writing, Opt. Lett. 30 (2005) 2248-2250.

[6] J. Burghoff, S. Nolte, A. Tünnermann, Origins of waveguiding in femtosecond laser-structured $\mathrm{LiNbO}_{3}$, Appl. Phys. A 89 (2007) 127-132.

[7] V. Apostolopoulos, L. Laversenne, T. Colomb, C. Depeursinge, R. Salathé M. Pollnau, R. Osellame, G. Cerullo, P. Laporta, Femtosecond-irradiationinduced refractive-index changes and channel waveguiding in bulk $\mathrm{Ti}^{3+}$. Sapphire, Appl. Phys. Lett. 85 (2004) 1122-1124.

[8] R. He, Q. An, Y. Jia, G.R. Castillo-Vega, J.R. Vázquez de Aldana, F. Chen, 
Femtosecond laser micromachining of lithium niobate depressed cladding waveguides, Opt. Mater. Exp. 3 (2013) 1378-1384.

[9] A.G. Okhrimchuk, V. Mezentsev, A. Shestakov, I. Bennion, Low loss depressed cladding waveguide inscribed in YAG: Nd single crystal by femtosecond laser pulses, Opt. Exp. 20 (2012) 3832-3843.

[10] H.D. Nguyen, A. Ródenas, J.R. Vázquez de Aldana, J. Martínez, F. Chen, M. Aguiló, M.C. Pujol, F. Díaz, Heuristic modelling of laser written mid-infrared $\mathrm{LiNbO}_{3}$ stressed-cladding waveguides, Opt. Exp. 24 (2016) 7777-7791.

[11] J. Hu, C. Menyuk, Understanding leaky modes: slab waveguide revisited, Adv. Opt. Phot. Rev. 1 (2009) 58-106.

[12] D.G. Lancaster, S. Gross, A. Fuerbach, H.E. Heidepriem, T.M. Monro M.J. Withford, Versatile large-mode-area femtosecond laser-written Tm: ZBLAN glass chip lasers, Opt. express 20 (25) (2012) 27503-27509.

[13] G.A. Torchia, A. Rodenas, A. Benayas, E. Cantelar, L. Roso, D. Jaque, Highly efficient laser action in femtosecond-written Nd:yttrium aluminum garnet ceramic waveguides, Appl. Phys. Lett. 92 (11) (2008) 111103.

[14] H. Liu, Y. Jia, J.R. Vázquez de Aldana, D. Jaque, F. Chen, Femtosecond laser inscribed cladding waveguides in Nd:YAG ceramics: fabrication, fluorescence imaging and laser performance, Opt. Exp. 20 (2012) 18620-18629.

[15] Yuechen Jia, J.R. Vazquez de Aldana, Oingming Lu, Daniel Jaque, Feng Chen, Enhanced second harmonic generation in femtosecond laser inscribed double-cladding waveguide of Nd:GdCOB crystal, J. Light. Technol. 31 (23) (2013) 3873-3878

[16] Jinman Lv, Yazhou Cheng, Qingming Lu, Javier R. Vázquez de Aldana, Xiaotao Hao, Feng Chen, Femtosecond laser written optical waveguides in zcut $\mathrm{MgO}: \mathrm{LiNbO}_{3}$ crystal: fabrication and optical damage investigation, Opt. Mater. 57 (2016) 169-173.

[17] Y. Jia, J.R. Vázquez de Aldana, C. Romero, Y. Ren, Q. Lu, F. Chen, Femtosecondlaser-inscribed $\mathrm{BiB}_{3} \mathrm{O}_{6}$ nonlinear cladding waveguide for second-harmoni generation, Appl. Phys. Exp. 5 (2012) 072701.

[18] H. Liu, Ch. Cheng C. Romero, J.R. Vázquez de Aldana, F. Chen, Graphene-based Y-branch laser in femtosecond laser written Nd:YAG waveguides, Opt. Exp. 23 (2015) 9730-9735.

[19] Ch. Cheng, C. Romero, J.R. Vázquez de Aldana, F. Chen, Superficial waveguide splitters fabricated by femtosecond laser writing of $\mathrm{LiTaO}_{3}$ crystal, Opt. Eng. 54 (2015) 067113.

[20] Hongliang Liu, Javier R. Vazquez de Aldana, Minghui Hong, Feng Chen, Femtosecond laser inscribed Y-Branch waveguide in Nd:YAG crystal: fabrication and continuous-wave lasing, IEEE J. Sel. Top. Quantum Electron. 22 (2) (2016) 227-230.

[21] M. Butt, H.D. Nguyen, A. Ródenas, C. Romero, P. Moreno, J.R. Vázquez de Aldana, M. Aguiló, R. Solé, M.C. Pujol, F. Díaz, Low repetition rate femtosecond laser writting of optical waveguides in KTP crystals: analysis of anisotropic refractive index changes, Opt. Exp. 23 (2015) 15343-15355.

[22] A. Rodenas, A. Benayas, J.R. Macdonald, J. Zhang, D.Y. Tang, D. Jaque, A.K. Kar, Direct laser writing of near-IR step-index buried channel waveguides in rare earth doped YAG, Opt. Lett. 36 (17) (2011) 3395-3397.

[23] J. Siebenmorgen, K. Petermann, G. Huber, K. Rademaker, S. Nolte, A. Tünnermann, Femtosecond laser written stress-induced $\mathrm{Nd}: \mathrm{Y}_{3} \mathrm{Al}_{5} \mathrm{O}_{12}$ (Nd: YAG) channel waveguide laser, Appl. Phys. B 97 (2009) 251-255.

[24] A. Siegman, Lasers, first ed., University Science Books, Sausalito, 1990.

[25] Y. Okamura, Sh. Yoshinaka, S. Yamamoto, Measuring mode propagation losses of integrated optical waveguides: a simple method, Appl. Opt. 22 (1983) 3892-3894.

[26] H. Liu, F. Chen, J.R. Vázquez de Aldana, D. Jaque, Femtosecond-laser inscribed double-cladding waveguides in Nd:YAG crystal: a promising prototype for integrated lasers, Opt. Lett. 38 (2013) 3294-3297. 